\title{
Diagnostic and prognostic characteristics of the enzyme linked immunosorbent rheumatoid factor assays in rheumatoid arthritis
}

\author{
Hendrik Visser, Luc B S Gelinck, Anna H Kampfraath, Ferdinand C Breedveld, \\ Johanna $M$ W Hazes
}

\begin{abstract}
Objective-To determine the diagnostic and prognostic test qualities of the enzyme linked immunosorbent assays (ELISA) for rheumatoid factor isotypes in rheumatoid arthritis (RA), and to compare them with the latex fixation test.

Methods-Rheumatoid factor tests were performed in 1988 consecutive new rheumatology outpatients within two months after their first visit to the outpatient clinic of the Department of Rheumatology of Leiden University hospital. The sensitivity, specificity, accuracy, and predictive values of the tests in discriminating $R A$ from non-rheumatoid arthritis and erosive from non-erosive disease after two years of follow up were determined and presented as receiver operating characteristic curves and posttest probability curves.
\end{abstract}

Results-The sensitivity of the ELISA for IgG, IgA, and IgM rheumatoid factor for RA versus all controls at optimal cut off titres was $72 \%, 44 \%$, and $69 \%$, respectively; the specificity was $52 \%, 84 \%$, and $86 \%$. For the latex fixation test the sensitivity was $66 \%$ and the specificity $91 \%$. The post-test probability of RA, at a clinical prevalence rate of $12 \%$, given a positive test result in the ELISAs for IgG, IgA, and IgM rheumatoid factor and the latex fixation test, was $17 \%, 27 \%, 40 \%$, and $49 \%$, respectively; with negative test results the probability was $7 \%, 8 \%, 5 \%$, and $5 \%$, respectively. The specificity of all tests in discriminating erosive from nonerosive RA at two years was low: $41 \%$, $44 \%, 47 \%$, and $58 \%$ for the ELISAs for IgG, IgA, and IgM rheumatoid factor and the latex fixation test, respectively.

Conclusion-The ELISAs for IgG and IgA rheumatoid factor are of no significance in diagnosing RA and in the prediction of erosive disease. The ELISA for IgM rheumatoid factor is a reasonable alternative for the latex fixation test when age and gender are taken in to consideration. The specificity of all rheumatoid factor tests in discriminating erosive from nonerosive RA is low.

(Ann Rheum Dis 1996; 55: 157-161)
The main role of rheumatoid factor in the clinical setting lies in its contribution to the diagnosis of rheumatoid arthritis (RA). The diagnostic value of the presence of rheumatoid factors has been studied most often for the classical latex fixation and Rose-Waaler agglutination assays $;^{1-6}$ however, little is known about the diagnostic properties of the enzyme linked immunosorbent assays (ELISAs) ${ }^{7}$ for quantitative detection of rheumatoid factor isotypes.

The results of rheumatoid factor tests are also used in making a prognosis in RA. In particular, the concentration of IgA rheumatoid factor is said to be useful in the prediction of bone erosions. ${ }^{8}$ There are considerable discrepancies, however, between the results of various studies concerning the prognostic value of rheumatoid factor isotypes. ${ }^{9-17}$

In January 1989, the ELISA for rheumatoid factor isotypes was introduced for routine rheumatoid factor testing in the Department of Rheumatology of the Leiden University Hospital, The Netherlands, which is the only referral clinic for rheumatic disorders in a district of approximately 300000 inhabitants. The latex fixation test remained in routine use as a standard until January 1992.

The objective of the present study was to determine the diagnostic and prognostic characteristics of the class specific ELISAs for rheumatoid factors and to compare them with those of the established latex fixation test.

\section{Patients and methods}

The medical records were reviewed of all patients $(n=1988)$ who first attended the outpatient clinic between January 1989 and October 1992, and who had blood removed for rheumatoid factor assays. Patients referred to the clinic for a second opinion were excluded. The following information was compiled from the medical charts: year of birth, gender, year of first visit to the outpatient clinic, time between onset of symptoms and first visit, time between first visit and first rheumatoid factor testing, first rheumatoid factor titres, clinical diagnosis, cumulative number of the revised 1987 American Rheumatism Association (ARA) criteria present, ${ }^{5}$ presence of typical bone erosions, ${ }^{518}$ time between first visit and onset of bone erosions, and period of clinical follow up. 
Table 1 Demographic characteristics of all patients and the two diagnostic subgroups, who had been newly referred to the outpatient rheumatology clinic and who had been tested for rheumatoid factors

\begin{tabular}{llll}
\hline & $\begin{array}{l}\text { All }(n=1988) \\
(100 \%)\end{array}$ & $\begin{array}{l}R A(n=235) \\
(11 \cdot 8 \%)\end{array}$ & $\begin{array}{l}\text { Non- } R A(n=1753) \\
(88 \cdot 2 \%)\end{array}$ \\
\hline Age (years) & $48(12-92)$ & $60(16-89)$ & $47(12-92)$ \\
Female (\%) & $67 \cdot 3$ & $63 \cdot 8$ & $67 \cdot 8$ \\
Duration of symptoms (months) & $12(0-576)$ & $6(0-576)$ & $12(0-480)$ \\
\hline
\end{tabular}

Values are median (range) where relevant.

All $=$ all patients; $\mathrm{RA}=$ rheumatoid arthritis; non- $\mathrm{RA}=$ all diseases other than rheumatoid arthritis.

All rheumatoid factor tests had been performed within two months after the first visit to the outpatient clinic. The class specific rheumatoid factors had been measured by an ELISA using mouse monoclonal antibodies against human IgG, IgA, and IgM, recognising a defined epitope on the Fc part of the immunoglobulins, together with the biotin-streptavidin enhancement system as described previously. ${ }^{7}$

In $34 \%$ of all eligible patients, rheumatoid factors had not been measured. Among this group, none had RA: $12 \cdot 3 \%$ were diagnosed as having other inflammatory rheumatic diseases, $77 \cdot 7 \%$ as having non-inflammatory rheumatic disease, and $10 \cdot 0 \%$ as having non-rheumatic disease.

The results of rheumatoid factor tests are usually taken into consideration when a clinical diagnosis of RA is made, which may lead to an overestimation of the diagnostic qualities of the tests. All analyses were therefore also performed using a diagnosis of RA based on the presence of four or more of the 1987 ARA criteria, ${ }^{5}$ excluding the rheumatoid factor criterion. On this basis, 91 patients were diagnosed as having RA, from 235 identified as having RA by the wider definition.

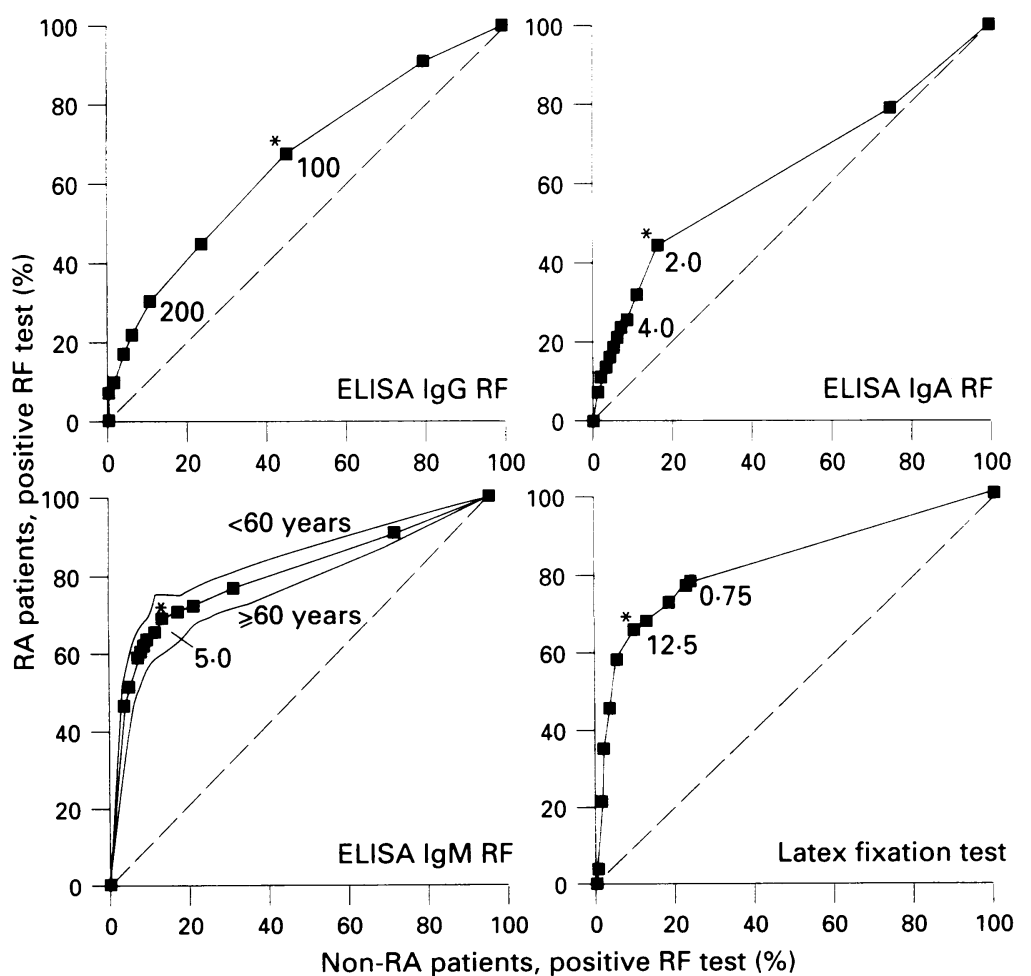

Figure 1 Receiver operating characteristic curves of the different enzyme linked immunosorbent assay (ELISA) rheumatoid factor $(R F)$ tests used to discriminate rheumatoid arthritis $(R A)(n=235)$ from non-rheumatoid arthritis (non- $R A)$

$(n=1753)$. The curves show the relation between true positive rate and false positive rate at different cut off titres. ${ }^{\star}$ Optimal cut off titre (IU).
The patients were stratified for age and gender to study the effect of these variables on rheumatoid factor positivity. For the analysis of the prognostic properties of the rheumatoid factor tests, we included only those RA patients who had been diagnosed as having RA according to the physician's opinion, whose disease was non-erosive at their first visit to the outpatient clinic, and who had been followed for at least two years $(n=62)$.

For all rheumatoid factor tests and their combinations at different cut off titres, the sensitivity, specificity, and accuracy (=sensitivity + specificity/2) in discriminating $\mathrm{RA}$ patients from non-RA patients were calculated. To assess the ability of the rheumatoid factor tests to predict the development of bone erosions, their sensitivity, specificity, and accuracy in discriminating erosive RA from non-erosive RA after two years of follow up were calculated at different cut off titres. The results were presented graphically using receiver operating characteristic (ROC) curves, plotting the relation between true positive rate (sensitivity, $\mathrm{y}$ axis) and false positive rate ( $1-$ specificity, $\mathrm{x}$ axis), for different cut off titres. ${ }^{19}$ Predictive values (=post-test probabilities) were calculated $^{20}$ and presented graphically plotting the pretest probability on the $\mathrm{x}$ axis and the posttest probability on the $y$ axis. The post-test probability represents the probability of a patient having RA, given a pretest probability (prevalence of RA) and the rheumatoid factor test result at a specific cut off titre.

\section{Results}

PATIENT CHARACTERISTICS

The patients were divided into two groups according to the diagnosis: group 1 consisted of those patients who had been diagnosed by the attending rheumatologist as having $\mathrm{RA}$ $(n=235)$; group 2 consisted of all other newly referred patients (non-RA) $(n=1753)$ comprising: patients with other inflammatory $(n=462)$ and non-inflammatory rheumatic disorders $(n=1138)$, and patients with nonrheumatic diseases $(n=153)$.

Table 1 displays the demographic characteristics of the patient population $(n=1988)$ and the diagnostic subgroups. The median age of the RA patients was 13 years greater than that of the non-RA patients, and the median time between onset of symptoms and first clinic visit was six months for RA patients but twice as long for non-RA patients. The prevalence rate of $\mathrm{RA}$ in all patients who first attended the outpatient clinic was $6 \%$; in the group of patients who had blood drawn for rheumatoid factor assays after selection by the physician it was $11 \cdot 8 \%$.

\section{DIAGNOSTIC CHARACTERISTICS}

The sensitivity and specificity of the rheumatoid factor assays in discriminating RA from non-RA patients are presented graphically for different cut off titres by means of ROC curves (fig 1). The curves for the ELISAs for both IgG and IgA rheumatoid factor showed poor test 


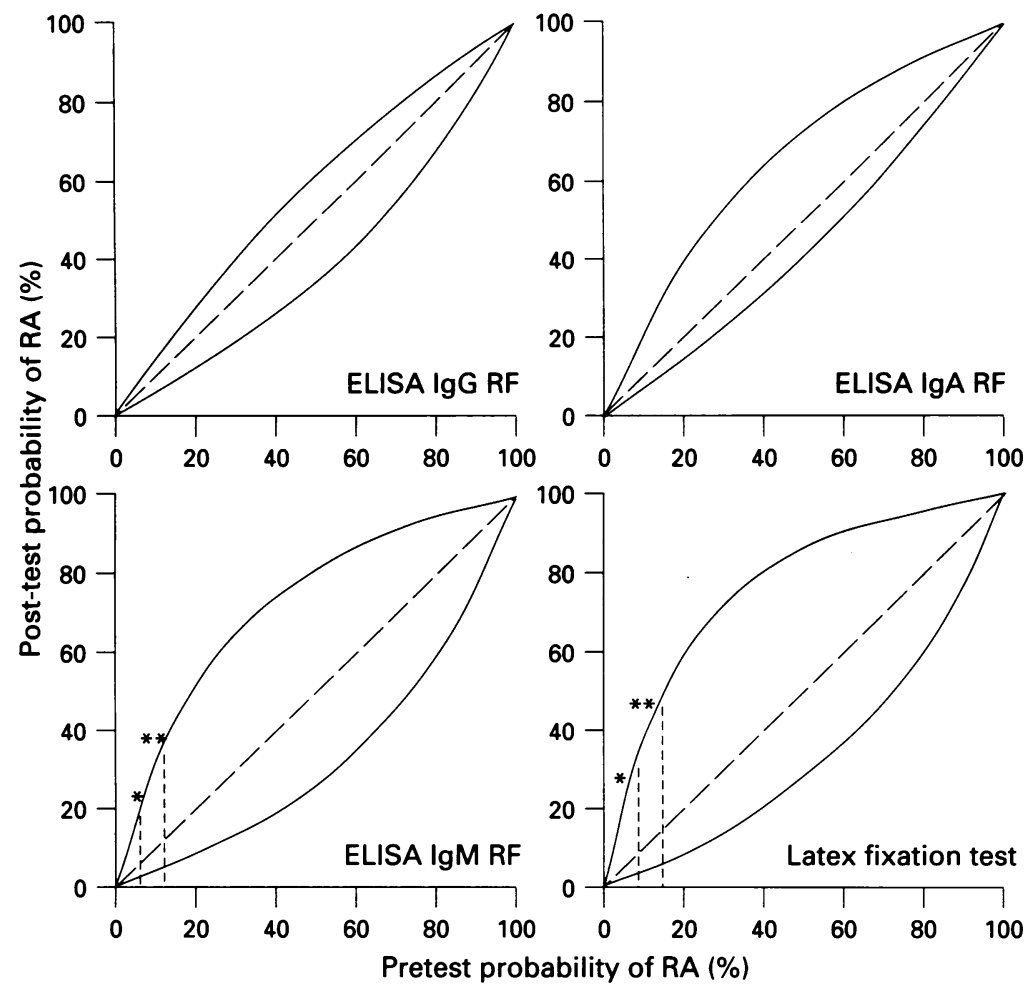

Figure 2 Post-test probability of rheumatoid arthritis $(R A)$ after a positive test result (upper bowed line) and after a negative test result (lower bowed line) at variable pretest probabilities (=prevalence), using the optimal cut off titre. Data for four different enzyme linked immunosorbent assay (ELISA) rheumatoid factor tests are shown. * Post-test probability of $R A$ at a clinical prevalence rate of $R A$ of $6.0 \%$ (before selection by the physician). ${ }^{\star *}$ Post-test probability of $R A$ at a clinical prevalence rate of $R A$ of $11 \cdot 8 \%$ (after selection by the physician).

characteristics at all cut off titres, as indicated by the position of the curves near the $45^{\circ}$ line. Both the ELISA for IgM rheumatoid factor and the latex fixation test showed much better test characteristics. The rheumatoid factor titres at which the accuracy of the tests was

Table 2 Diagnostic characteristics of the enzyme linked immunosorbent assay (ELISA) rheumatoid factor (RF) tests and the latex fixation test (LFT) at optimal cut off titres, in discriminating rheumatoid arthritis from non-rheumatoid arthritis $(n=1753)$

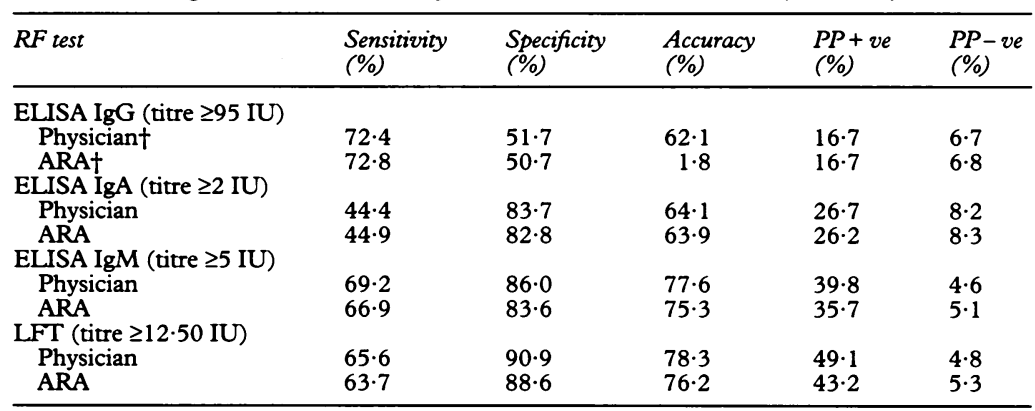

tRheumatoid arthritis diagnosed according to the physician's opinion $(n=235)$ or the presence of more than four 1987 American Rheumatism Association (ARA) criteria, excluding the rheumatoid factor criterion $(n=91)$.

$\mathrm{PP}+\mathrm{ve}=$ Post-test probability of rheumatoid arthritis after a positive RF test; $\mathrm{PP}-\mathrm{ve}=$ post-test probability of rheumatoid arthritis after a negative RF test; predictive values calculated at a prevalence rate of rheumatoid arthritis of $11.8 \%$.

Table 3 Prevalence of increased rheumatoid factors in the different subgroups of patients at optimal cut off titres

\begin{tabular}{lllll}
\hline$R F$ test & $\begin{array}{l}R A(\%) \\
(n=235)\end{array}$ & $\begin{array}{l}I R D(\%) \\
(n=462)\end{array}$ & $\begin{array}{l}\text { Non-IRD (\%) } \\
(n=1138)\end{array}$ & $\begin{array}{l}\text { Other (\%) } \\
(n=153)\end{array}$ \\
\hline ELISA IgG (titre $\geq 95$ IU) & $72 \cdot 4$ & $53 \cdot 4$ & $46 \cdot 2$ & $48 \cdot 7$ \\
ELISA IgA (titre $\geq 2$ IU) & $44 \cdot 4$ & $16 \cdot 5$ & $15 \cdot 9$ & $18 \cdot 2$ \\
ELISA IgM (titre $\geq 5$ IU) & $69 \cdot 2$ & $14 \cdot 1$ & $13 \cdot 2$ & $20 \cdot 1$ \\
LFT (titre $\geq 12 \cdot 50$ IU) & $65 \cdot 6$ & $10 \cdot 3$ & $8 \cdot 0$ & $14 \cdot 3$ \\
\hline
\end{tabular}

RA = Rheumatoid arthritis; IRD = other inflammmatory rheumatic disorders; Non-IRD = noninflammatory rheumatic disorders; others = patients with non-rheumatic diseases; $R F=$ rheumatoid factor; ELISA = enzyme linked immunosorbent assay; LFT = latex fixation test. greatest were chosen as optimal cut off titres. At the optimal cut off titre of 5.0 IU, the sensitivity of the ELISA for IgM rheumatoid factor was $69 \cdot 2 \%$ and the specificity $86.0 \%$. For the latex fixation test the sensitivity was $65.6 \%$ and the specificity $90.9 \%$ at the optimal cut off titre of $12 \cdot 50 \mathrm{IU}$.

Both sensitivity and specificity, in particular of the ELISA for IgM, decreased with advancing age of the patient. Figure 1 shows the ROC curves of the ELISA for IgM for two age groups of patients. For the latex fixation test the two curves almost overlapped. When the patients were stratified for gender, all rheumatoid factor tests were more sensitive in men than in women (data not shown).

The predictive values of both the ELISA for IgG and that for IgA rheumatoid factor in RA diagnosis were low, with a poor gain from pretest to post-test probability of RA (fig 2), but the predictive values of both the ELISA for IgM and the latex fixation test for the diagnosis of RA were much greater (fig 2). Table 2 summarises the test characteristics of the different rheumatoid factor tests at optimal cut off titres for RA versus non-RA patients. In general, the way in which RA was defined did not substantially change the test characteristics. The test characteristics of combinations of the different ELISA rheumatoid factor tests were not better than the test characteristics of the ELISA for IgM or the latex fixation test alone (data not shown). Table 3 shows the prevalence of increased rheumatoid factors at optimal cut off titres in the four different subgroups of patients.

\section{PROGNOSTIC CHARACTERISTICS}

For the analysis of the prognostic properties of the rheumatoid factor tests, $62 \mathrm{RA}$ patients were included whose disease was non-erosive at their first clinic visit and who had been followed for at least two years. Seventeen patients with the same follow up time were excluded because they had erosions at the initial presentation. After two years of follow up, 30 patients had developed bone erosions, whereas 32 patients still had non-erosive disease. The erosive and non-erosive groups did not differ significantly in gender, or in age of the patient at first clinic visit. The median time between symptom onset and the first visit was slightly longer in the patients who developed erosions than in those who did not: 6.5 months (range 1-72) and $6 \cdot 0$ months (range 1-60), respectively.

The sensitivity and specificity of the rheumatoid factor tests performed within two months after the first clinic visit, in discriminating erosive from non-erosive RA after two years of follow up, are presented graphically for different cut off titres by means of ROC curves (fig 3). The test characteristics of the ELISAs for both IgG and IgA rheumatoid factor were poor; those of the ELISA for IgM rheumatoid factor and the latex fixation test were better, though the latter performed best: at the optimal cut off titre of 25.0 IU the sensitivity of the latex fixation test was $83.3 \%$ and the specificity $58 \cdot 1 \%$ (fig 3 ). 


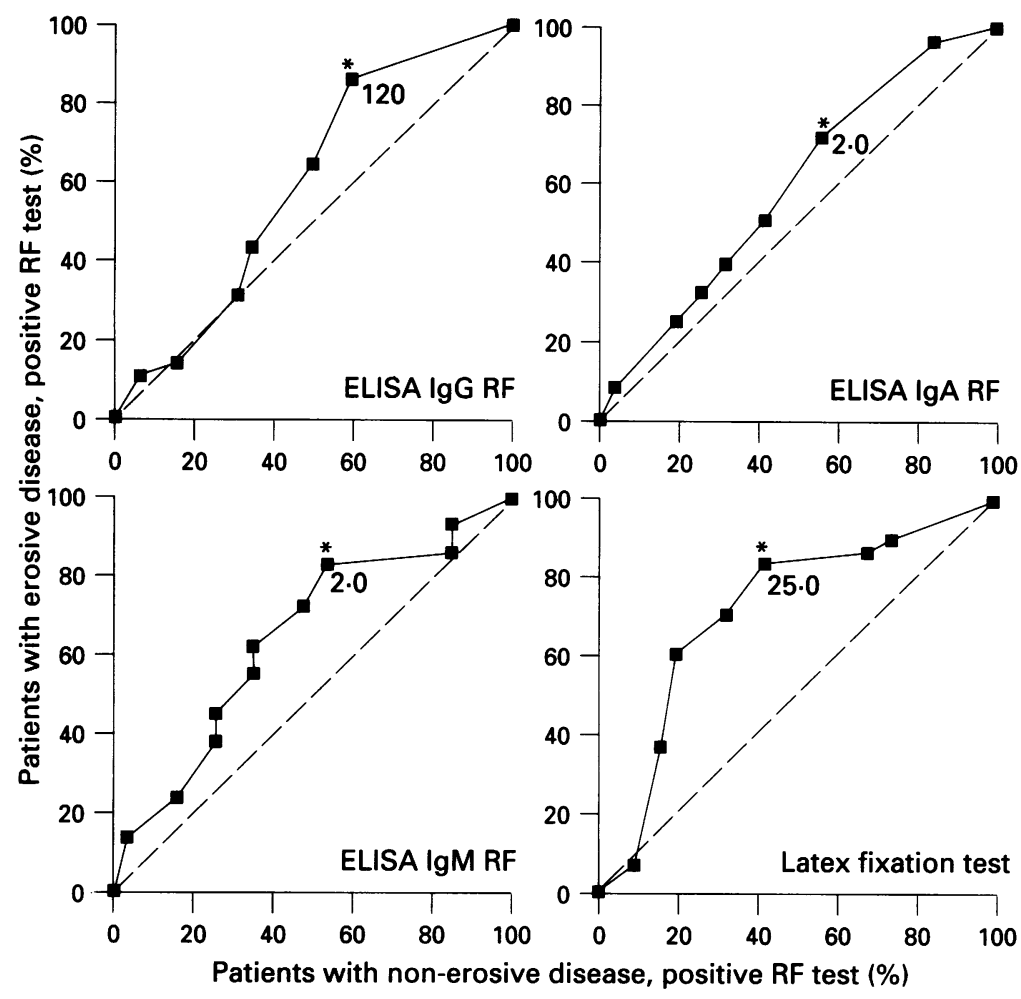

Figure 3 Receiver operating characteristic curves of the different enzyme linked immunosorbent assay (ELISA) rheumatoid factor (RF) tests when performed within two months after the first visit to the clinic to discriminate erosive from non-erosive rheumatoid arthritis after two years of follow up. The curves show the relation between true positive and false positive rate at different cut off titres. ${ }^{\star}$ Optimal cut off titre (IU).

\section{Discussion}

The present study has shown that the diagnostic characteristics of the ELISAs for both IgG and IgA rheumatoid factor in discriminating RA from non-RA are poor. The test characteristics of the ELISA for IgM rheumatoid factor and the latex fixation test proved to be much better, and compared reasonably well to each other, though the specificity of the latex fixation test was greater than that of the ELISA for IgM, resulting in a greater predictive value for a diagnosis of RA.

These results are partly in accordance with those of a previous study ${ }^{6}$ in which the latex fixation test was found to be a very specific test. The greater sensitivity and specificity of the latex fixation test found in that study can be explained by differences in patient selection, criteria for the diagnosis of RA, and study design. In an earlier Dutch study, ${ }^{7}$ a high specificity of the ELISA for all three rheumatoid factor isotypes was found. The selection of patients and controls, however, did not reflect clinical practice. The patients in that study were known to have had definite RA for some time, whereas patients in our study had early $\mathrm{RA}$ and were in a diagnostic phase; furthermore, selected patients with known systemic lupus erythematosus, ankylosing spondylitis, osteoarthritis, and bronchial asthma comprised the controls in the earlier study, but in the present study the controls consisted of all non-RA patients who first attended the outpatient clinic. The design of the present study, therefore, was more appropriate to the determination of the diagnostic qualities of the rheumatoid factor test. In an intervening study, ${ }^{21}$ a much lower specificity of the ELISA rheumatoid factor test was found when controls were selected randomly from the general population.

In that study also, the prevalence of the various rheumatoid factor isotypes in the control population seemed to vary with the age of the patient: IgM rheumatoid factor increased, and IgG rheumatoid factor decreased, with age. ${ }^{21}$ In the present study, we found that the sensitivity and specificity of the ELISA for IgM decreased with advancing age of the patient, resulting in a greater diagnostic power of the ELISA for IgM in the younger age groups. For the latex fixation test, this effect of age was minimal. All rheumatoid factor assays had a greater sensitivity in men with RA compared with women patients, which is in accordance with the findings of other studies. ${ }^{6} 22$

The diagnostic characteristics of the rheumatoid factor tests are in part dependent on the characteristics of the clinical population in which the test is used, such as the criteria for the definition of RA, age, gender, and the presence of other rheumatoid factor related diseases. ${ }^{6} 2122$ The test characteristics found in the present study will therefore not necessarily be in accordance with those observed in other clinic populations. However, the ratio of the diagnostic qualities of the different rheumatoid factor tests is not affected by these population characteristics, so that the IgM rheumatoid factor test can be accepted as being superior to the IgA and IgG tests.

With regard to the prediction of erosions at two years, the discriminating qualities of the latex fixation test appeared to be better than those of the ELISAs for rheumatoid factor isotypes. In particular, the test characteristics of the ELISAs for IgG and IgA rheumatoid factor were poor. The specificity of all tests was low. In the literature, the results of cross sectional and longitudinal studies on the association between rheumatoid factor isotypes and the development of bone erosions are inconsistent. ${ }^{9-17}$ This inconsistency may result from several factors such as differences in patient selection, study design, and the techniques used to measure the rheumatoid factor isotypes. In one study, only RA patients with negative agglutination assays were included. ${ }^{12}$ The mean time between the onset of symptoms and the time of the first rheumatoid factor assay varied in the different studies and was sometimes more than two years. ${ }^{11} 17$ Furthermore, the follow up time in the various prospective studies varied from two to 10 years. In a Scandinavian study, ${ }^{13}$ only radiographs of the hands were obtained, in contrast with most other studies in which radiographs of both hands and feet were assessed. In the present study, the rheumatoid factor tests were performed in all patients within two months after their first visit to the outpatient clinic, and radiographs of both hands and feet were assessed. Because of the limited time of follow up, no assessment of the severity of bone erosions could be made, which allowed us to discriminate only between erosive and nonerosive disease. A follow up period of two years 
is usually sufficient for the development of erosive disease. ${ }^{23}$

In conclusion, the results of this study suggest that the diagnostic and prognostic qualities of the ELISAs for IgG and IgA rheumatoid factors for RA are poor, while the diagnostic qualities of the ELISA for IgM are much better, and comparable to those of the latex fixation test. When the ELISA for IgM is used as diagnostic test for RA, the influence of age and gender on rheumatoid factor positivity should be taken into consideration. Compared with the latex fixation test, the ELISA for rheumatoid factors is more reproducible, costs less, and is time saving, especially in larger clinics where large numbers of serum samples can be processed at the same time. In such a setting, therefore, the ELISA for IgM can replace the latex fixation test as the diagnostic test. The prognostic qualities of the latex fixation test are better than those of the Elisa for IgM, but still not good enough to provide guidelines for decisions concerning treatment.

1 Egeland T, Munthe E. Rheumatoid factors. Clin Rheum Dis 1983; 9: 135-60

2 Aho K Tuomi T, Heliövaara M, Palosuo T. Rheumatoid factors and rheumatoid arthritis. Scand $\mathcal{F}$ Rheumatol 1988; 74 (suppl): 41-4.

3 Aho K, Palosuo T, Raunio V, Puska P, Aromaa A Solonen J T. When does rheumatoid disease start? Arthritis Rheum 1985; 28: 485-9.

4 Valkenburg H A, Ball J, Burch T A, Bennett P H, Lawrence J S. Rheumatoid factors in a rural population. Ann Rheum Dis 1966; 25: 497-508.

5 Amett F C, Edworthy S M, Bloch D A, et al. The American Rheumatism Association 1987 revised criteria for the classification of rheumatoid arthritis. Arthritis Rheum 1988; 31: 315-24.

6 Wolfe F, Cathey M A, Roberts F K. The latex test revisited. Rheumatoid factor testing in 8,287 rheumatic disease patients. Arthritis Rheum 1991; 34: 951-60.

7 Otten H G, Daha M R, de Rooij H H, Breedveld F C. Quantitative detection of class-specific rheumatoid factors using mouse monoclonal antibodies and the biotin/streptavidin enhancement system. $\mathrm{Br} \mathcal{F}$ Rheumatol 1989; 28: 310-6.
8 Jónsson T, Valdimarsson $\mathrm{H}$. Is measurement of rheumatoid factor isotypes clinically useful? Ann Rheum Dis 1993; 52: $161-4$

9 Tarkowski A, Nilsson L A. Isotype-specific measurement of rheumatoid factor with reference to clinical features of rheumatoid arthritis. F Clin Lab Immunol 1983; 12: 129-35.

10 Gioud-Paquet $M$, Auvinet $M$, Raffin $T$, et al. IgM rheumatoid factor (RF), IgA RF, IgE RF and IgG RF detected by ELISA in rheumatoid arthritis. Ann Rheum Dis 1987; 46: 65-71.

11 Arnason J A, Jonsson T, Brekkan A, Sigurjonsson K, Valdimarsson $H$. Relation between bone erosions and rheumatoid factor isotypes. Ann Rheum Dis 1987; 46: $380-4$.

12 Eggelmeijer F, Otten H G, de Rooy H H, Daha M R, Breedveld F C. Significance of rheumatoid factor isotypes in seronegative rheumatoid arthritis. Rheumatol Int 1990; 10: 43-6.

13 Teitsson I, Withrington $R H$, Seifert $M H$, Valdimarsson $H$. Prospective study of early rheumatoid arthritis. I. Prognostic value of IgA rheumatoid factor. Ann Rheum Dis 1984; 43: 673-8.

14 Winska Wiloch $\mathrm{H}$, Thompson $\mathrm{K}$, Young A, et al. IgA and IgM rheumatoid factors as markers of later erosive changes in rheumatoid arthritis (RA). Scand $\mathcal{F}$ Rheumatol 1988; 75 (suppl): $238-43$.

15 Möttönen $T$, Hannonen $P$, Jokinen I, Arvilommi $M$, Oka M. Relation between bone erosions and rheumatoid factor IgA and IgM isotypes in recent onset rheumatoid arthritis. Scand $\mathcal{F}$ Rheumatol 1988; 75 (suppl): 244-9.

16 Eberhardt K B, Svensson B, Truedsson L, Wollheim F A. The occurrence of rheumatoid factor isotypes in early definite rheumatoid arthritis. No relationship with erosions or disease activity. $f$ Rheumatol 1988; 15: $1070-4$.

17 Zeben van D, Hazes J M W, Zwinderman A H, Cats A, van der Voort E A M, Breedveld F C. Clinical significance of rheumatoid factors in early rheumatoid arthritis: results of a follow up study. Ann Rheum Dis 1992; 51: results of

18 Paimela L. The radiographic criterion in the 1987 revised criteria for rheumatoid arthritis. Reassessment in a prospective study of early disease. Arthritis Rheum 1992; 35: $255-8$.

19 The use of diagnostic information to revise probabilities. In: Weinstein M C, Fineberg $\mathrm{H}$ V, eds. Clinical decision analysis. Philadelphia: W B Saunders, 1980; 114-27.

20 Ahlbom A, Norell S. Introduction to modern epidemiology. Stockholm: Epidemiology Resources Inc, 1984; 23-8.

21 Schaardenburg van D, Lagaaij A M, Otten H G, Breedveld F C. The relation between class-specific serum rheumatoid factors and age in the general population. $\mathrm{Br}$ rheumatoid factors and age in

22 Cats A, Klein F. Quantitative aspects of the latex-fixation and Waaler-Rose tests. Ann Rheum Dis 1970; 29: 663-72.

23 Brook A, Corbett M. Radiographic changes in early rheumatoid disease. Ann Rheum Dis 1977; 36: 71-3. 